\title{
SOLPS-ITER modelling of ITER edge plasma with drifts and currents
}

E. Kaveeva ${ }^{1 *}$, V. Rozhansky ${ }^{1}$, I. Senichenkov ${ }^{1}$, E. Sytova ${ }^{1-5}$, I. Veselova ${ }^{1}$, S. Voskoboynikov ${ }^{1}$, X. Bonnin ${ }^{2}$, R. A. Pitts ${ }^{2}$, A.S. Kukushkin ${ }^{6,7}$, S. Wiesen ${ }^{8}$, D. Coster ${ }^{3}$

${ }^{1}$ Peter the Great St.Petersburg Polytechnic University, Polytechnicheskaya 29, 195251

St.Petersburg, Russia

${ }^{2}$ ITER Organization, Route de Vinon-sur-Verdon, CS 90 046, 13067 St Paul Lez Durance Cedex, France;

${ }^{3}$ Max-Planck Institute for Plasma Physics, D-85748 Garching, Germany;

${ }^{4}$ Ghent University, Department of Applied physics, Ghent, Belgium;

${ }^{5}$ Aix-Marseille Universite, Ecole Doctorale Physique et Sciences de la Matiere, Marseille, France;

${ }^{6}$ NRC Kurchatov Institute, Kurchatov sq. 1, 123182 Moscow, Russia;

${ }^{7}$ NRNU MEPhI, Kashirskoye sh. 31, 115409 Moscow, Russia;

${ }^{8}$ Forschungszentrum Jülich GmbH, Institut für Energie- und Klimaforschung - Plasmaphysik, D-

52425 Jülich, Germany

*Corresponding author e-mail: kaveeva@spbau.ru

\begin{abstract}
Using the new version of the SOLPS plasma boundary code package, SOLPS-ITER, the paper presents the first ever simulations of the ITER burning baseline H-mode edge plasma with drifts and currents activated. Neon $(\mathrm{Ne})$ seeded discharges for divertor power dissipation are considered. The results for divertor and scrape-off layer (SOL) parameters with and without drifts are compared, both for the SOLPS-ITER simulations and against the earlier SOLPS-4.3 modelling (which did not include a drift description) constituting the bulk of the existing ITER divertor simulation database. Whereas the drift effect on the equatorial midplane (main chamber) density and temperature profiles is moderate, drifts increase the peak heat flow to the outer divertor target. This effect is more pronounced for regimes with low sub-divertor neutral pressure, when even drift-free SOLPS4.3 simulations find strong out-in target power asymmetries. An important conclusion is thus that if ITER operates as expected with partially detached divertor targets, drifts should not influence the power handling, but that in the case of divertor reattachment, they will act to worsen the target loading, increasing the need for vigilance in detachment control. Comparing SOLPS-4.3 and SOLPS-ITER results for the key peak target heat flux versus sub-divertor neutral pressure operating domain, SOLPS-ITER with drifts predicts a narrower operational window for the divertor pressure.
\end{abstract}

Keywords: SOLPS-ITER; drifts; ITER divertor plasma; asymmetries 


\section{Introduction}

Edge plasma performance and divertor power handling are the key issues for successful operation of ITER. Plasma boundary simulations with the SOLPS code (v.4.3) have until recently been the main physics input to the ITER divertor design [1]. The SOLPS4.3 code suite includes a Braginskii model for parallel transport (the fluid code B2), an experiment-based empirical description of anomalous transport, and a comprehensive kinetic neutral Monte-Carlo model (the EIRENE code). However, it contains no description of fluid drifts [1,2], whereas experience of modeling existing tokamaks $[3,4]$ as well as many experiments (see e.g. [5,6,7]) show that the impact of the drifts on the boundary plasma parameters can be significant. In 2015, a new, updated version of the SOLPS package was released by the ITER Organization in the form of the SOLPS-ITER code [8,9], which includes drifts and currents in the fluid model (B2.5), using the formulation developed for SOLPS5.2 [3], which has been deployed for the simulation of many existing devices $[3,4,10]$. Although several amendments have since been made to improve the convergence and numerical accuracy required for simulation at the ITER scale [11], the general physical features of the drift model have not changed in SOLPS-ITER. The new code has already been used for drift modeling of ASDEX-Upgrade [12], C-Mod [13] and DIII-D [14]. This paper presents the first ever results obtained with SOLPS-ITER with full drifts and currents activated for the ITER baseline burning plasma with neon $(\mathrm{Ne})$ impurity seeding, including comparisons with code runs without drifts and with the earlier SOLPS-4.3 results, which by default do not include a drift description.

\section{Modeling}

\subsection{Modeling setup}

Simulations are performed for a fuel throughput scan (corresponding to a scan in sub-divertor neutral pressure) under baseline, full performance $\left(\mathrm{Q}_{\mathrm{DT}}=10\right)$ conditions on ITER with power into the scrapeoff layer, $\mathrm{P}_{\mathrm{SOL}}=100 \mathrm{MW}$ and with moderate Ne seeding for divertor power dissipation. The EIRENE particle reflection model corresponds to all-metal plasma-facing components (PFC) covered by beryllium $(\mathrm{Be})$ everywhere; that is, the tungsten $(\mathrm{W})$ divertor targets are assumed to be covered by $\mathrm{Be}$ deposition (from main chamber wall erosion) for simplicity. This is the same approach adopted for the existing SOLPS-4.3 simulation database $[15,16]$. Neither Be nor W impurity are included in the model. Beryllium is a weak radiator and taking its sputtering into account would not change the results significantly [15]. Tungsten radiates strongly only at electron temperatures, $T_{e}$ much higher than expected in the SOL, so that if $\mathrm{W}$ radiation were to be significant in the edge, the core radiation would be excessive and incompatible with burning plasma operation. Very recent SOLPS-ITER modelling of the low power, non-nuclear phases of ITER operation has shown that the choice of divertor material (W or Be-coating of $\mathrm{W}$ surfaces) does not change the overall simulations results for detachment behavior, at least in the absence of drifts [17].

The input data for the simulations are taken from a reference SOLPS4.3 case [18], in which both targets are partially detached. Full recycling is imposed at all PFCs, with the exception of the surface below the divertor dome, where the albedo is set to 0.9928 to represent the appropriate divertor cryo-pumping speed $[16,18]$. Main chamber gas puffing (which controls the detachment state) is imposed as for the original SOLPS-4.3 runs (see [17] for a discussion of the impact of gas puffing location, which does 
not influence the main results). Outflows from the core region are set at $1.0 \cdot 10^{22}$ particles $/ \mathrm{s}$ for deuterium (D) and $1.0 \cdot 10^{20}$ particles/s for helium (corresponding respectively to pellet fueling and fusion alpha production). Note that tritium species are not distinguished in the simulations, also in common with [18]. The simulation domain and the pumping and puffing positions are shown in Fig.1(a).

Two main Ne-seeded SOLPS-ITER scenarios are considered (with and without drifts), with a low and 2.6x higher rate of D puffing. The parameters of these scenarios are compiled in Table 1. Additional runs have been made to complete the fuel throughput scan to establish the scaling of divertor heat load with neutral pressure (see Section 2.5). The peak power loading of the divertor targets, $\mathrm{q}_{\mathrm{pk}}$ listed in the table includes the plasma and neutral energy flow (the latter calculated in EIRENE), recombination on the surface and radiation from the plasma. It assumes cylindrically symmetric targets and hence does not include divertor component shaping (see [16] for a detailed discussion of the impact of shaping). The average $\mathrm{Ne}$ concentration at the separatrix, $\mathrm{c}_{\mathrm{Ne}}$ is defined as the ratio of the surface averaged values of the $\mathrm{Ne}$ and $\mathrm{D}$ densities along the first closed flux surface outside the separatrix, see Fig.1(a) and [16]. These values can differ from the ratio of densities at the outer midplane (omp) due to significant poloidal variations of the Ne density along the flux surface. Along with $\mathrm{q}_{\mathrm{pk}}$, the divertor neutral pressure, $\mathrm{p}_{\mathrm{n}}$ is a key divertor operating parameter [16]. The latter, also included in Table 1, is defined as the average value along the two private flux region (PFR) boundaries of the B2.5 mesh (see Fig. 1a). Note that for the higher $\mathrm{p}_{\mathrm{n}}$ cases (2a,b in Table 1), the fuel throughput is about a factor 2 higher than the maximum peak stationary throughput of which ITER will be capable. This is not a major issue given that the value of $\mathrm{p}_{\mathrm{n}}$ is more important for divertor operation than the throughput $[19,20]$. The same operational parameters in the SOLPS4.3 modeling could be recovered (except for He exhaust) by reducing throughput and pumping speed.

The computational time required for a single SOLPS-ITER simulation at the ITER scale on the St. Petersburg Polytechnic University cluster (56 processors Intel Xeon CPU E5-2697 v3 @ 2.60GHz) ranges from a week to more than a month, depending on the discharge parameters, impurity species, and initial state from which the numerical convergence starts. Simulations with drifts require the application of speed-up schemes [11] for the runs to be at all practicable. An artificial anomalous electric conductivity was added to the equations for numerical reasons to provide convergence [3], though it was chosen sufficiently small to have no impact on the simulated electric field.

The anomalous radial transport coefficients are shown in Fig.1(b). In the SOL they are set at the same values as those used in the reference SOLPS-4.3 simulations. This leads to almost the same distribution of the electron heat flow towards the divertor from the SOL, Fig.1(c), and therefore the same SOL parallel heat flux density width $\lambda_{\mathrm{q}} \sim 3-4 \mathrm{~mm}$. As discussed at length in [16], although this value of $\lambda_{\mathrm{q}}$ is much larger than that expected from the most recent experimental scaling derived from inter-ELM H-modes on current tokamaks [21], it is slightly lower than the recent predictions obtained with the XGC1 and BOUT++ codes for the ITER burning plasma at 15 MA plasma current[22]. In the confined plasma region, the expected ITER H-mode conditions are reproduced by reducing the transport coefficients in the transport barrier region limited by the separatrix and the flux surface $5 \mathrm{~cm}$ into the core at the omp. This is the same approach which has been adopted in the SOLPS modeling of existing machines [3,4], Fig.1(b). 
Table 1. Main parameters of modelled scenarios. The table columns contain: the scenario description; the deuterium puffing rate; the neon seeding rate; the neutral pressure in the divertor taken at the private flux region (PFR) boundary of the B2.5 mesh (see Fig.1(a)); the peak power loading of the outer and inner divertor targets; the relative Ne concentration, separatrix-averaged; the power radiated from the outer and inner divertor regions integrated below the X-point, including the corresponding PFR regions (the values in parentheses give the radiation due to partially ionized impurities - $\mathrm{Ne}$ and $\mathrm{He}$ in these simulations). The simulation run numbers in Column 1 are those which identify the code runs in the ITER Integrated Modelling Analysis Suite (IMAS).

\begin{tabular}{|c|c|c|c|c|c|c|c|c|}
\hline $\begin{array}{c}\text { Scenario/IMAS } \\
\text { number }\end{array}$ & $\begin{array}{c}\text { D puff., } \\
\text { particles/s }\end{array}$ & $\begin{array}{c}\text { Ne puff., } \\
\text { particles/s }\end{array}$ & $\begin{array}{c}\mathrm{p}_{\mathrm{n}}, \\
\mathrm{Pa}\end{array}$ & $\begin{array}{c}\mathrm{q}_{\mathrm{pk}} \text { outer } \\
\mathrm{MW} / \mathrm{m}^{2}\end{array}$ & $\begin{array}{c}\mathrm{q}_{\mathrm{pk}} \text { inner } \\
\mathrm{MW} / \mathrm{m}^{2}\end{array}$ & $\begin{array}{c}\mathrm{c}_{\mathrm{Ne}} \\
\%\end{array}$ & $\begin{array}{c}\mathrm{P}_{\text {rad,in, }} \\
\mathrm{MW}\end{array}$ & $\begin{array}{c}\mathrm{P}_{\text {rad,in, }}, \\
\mathrm{MW}\end{array}$ \\
\hline $\begin{array}{c}\text { 1a, drifts off } \\
\# 123008\end{array}$ & $0.75 \cdot 10^{23}$ & $0.55 \cdot 10^{20}$ & 5.1 & 5.53 & 6.14 & 1.4 & $\begin{array}{c}33.7 \\
(28.3)\end{array}$ & $\begin{array}{c}12.8 \\
(7.4)\end{array}$ \\
\hline $\begin{array}{c}\text { 1b, drifts on } \\
\# 123014\end{array}$ & $0.75 \cdot 10^{23}$ & $0.55 \cdot 10^{20}$ & 5.0 & 11.95 & 6.12 & 1.3 & $\begin{array}{c}9.9 \\
(6)\end{array}$ & $\begin{array}{c}23.4 \\
(17.7)\end{array}$ \\
\hline $\begin{array}{c}\text { 2a, drifts off } \\
\# 123010\end{array}$ & $1.95 \cdot 10^{23}$ & $0.5 \cdot 10^{20}$ & 10.8 & 6.12 & 6.54 & 0.33 & $\begin{array}{c}29.9 \\
(21.1)\end{array}$ & $\begin{array}{c}16 \\
(7.1)\end{array}$ \\
\hline $\begin{array}{c}\text { 2b,drifts on } \\
\# 123018\end{array}$ & $1.95 \cdot 10^{23}$ & $0.5 \cdot 10^{20}$ & 10.7 & 6.28 & 6.04 & 0.52 & $\begin{array}{c}27.1 \\
(19.4)\end{array}$ & $\begin{array}{c}17.4 \\
(8)\end{array}$ \\
\hline
\end{tabular}

\subsection{Electrostatic potential}

The electrostatic potential distribution, which can only be properly calculated when drifts are included in the simulations, is shown in Fig. 2 for Case \#2b in Table 1. Similarly to the result previously found for SOLPS-ITER drift modelling of partially detached H-modes on ASDEX Upgrade [12], the potential peaks in the X-point vicinity. The origin of this maximum is the same as in [12], namely the parallel current arising to short circuit the $\nabla \mathrm{B}$ drift. Analysis of the electron parallel momentum balance, Fig. 3(a), shows that in the cold PFR, where the electric conductivity is low, the electric field is determined by the parallel currents closing the current due to the grad-B drift and is directed from the top of the PFR to the divertor targets. Fig. 3(a) shows the momentum balance at the inner side of PFR. At the outer side the balance is qualitatively similar. In the main part of the SOL, the electric force compensates the electron pressure gradient and the thermal force, whereas the contribution of ion-electron friction (which is proportional to the current) is negligible, Fig. 3(b). As a result, the electric field in the SOL is of the "Boltzmann" type, $\varphi \sim T_{e} / e$ whereas in the PFR, the potential can be much higher, $\varphi>10 T_{e} / e$.

In the confined region, the radial electric field, Fig.4 is close to its neoclassical value computed analytically according to the expression in [3], which is typical for simulations with SOLPS5.2 for existing tokamaks [3] and for ITER [23]. For Case \#1b at lower divertor pressure and lower separatrix density, the radial electric field in the pedestal region is stronger due to the larger neoclassical contribution associated with the density gradient, Fig. 5(a). 


\subsection{Impact of drifts on the SOL and divertor plasma parameters}

Figure 5(a-c) compiles omp radial profiles of $\mathrm{T}_{\mathrm{e}}$, main ion density, $\mathrm{n}_{\mathrm{D}}$ and total $\mathrm{Ne}$ density $\mathrm{n}_{\mathrm{Ne}}$ summed over all charge states for the 4 cases in Table 1 (low and high throughput, drifts on and off). Independent of fuel throughput, the principal $\mathrm{Ne}$ charge state contributor is $\mathrm{Ne}^{+8}$ in the SOL and the fully ionized state $\mathrm{Ne}^{+10}$ in the core. For the single Case \#2b, Fig. 5(d) compiles the density profiles for the individual $\mathrm{Ne}$ charge states.

At low throughput, $\mathrm{T}_{\mathrm{e}}$ and $\mathrm{n}_{\mathrm{D}}$ in the $\mathrm{H}$-mode pedestal region vary only slightly when drifts are switched on. There is essentially no change to the profiles when drifts are activated for Case \#2 at higher throughput. The plasma feature most sensitive to changing fuel gas puffing rate or activation of drifts is the Ne distribution. At the omp the total Ne density profiles are markedly different for Case \#1 with and without drifts, but the impact on the omp plasma parameters is rather modest. Section 2.4 discusses the origin of the Ne redistribution in the SOL when drifts are switched on. In the divertor, the redistribution of $\mathrm{Ne}$, as the main radiating component, leads in Case \# to a significant change of the whole solution. In the omp SOL, the $T_{e}$ profiles are almost identical for all four simulations, indicating that the SOL widths for the electron temperature and the electron parallel heat flow are the same for the divertor neutral pressures chosen, either with or without drifts, Fig. 5(e).

Figure 6 is the analog to Fig. 5 but now at the divertor targets, with the profiles plotted as a function of distance along the target relative to the strike point position. The Ne density in the divertor (summed over all charged states) is about three orders of magnitude lower than that of the main ions, while at the omp, it is lower by two orders of magnitude only, showing moderate retention of Ne in the outer divertor. This tendency is illustrated in Fig. 6(i,j) which compares the ratios of concentrations of impurity at the plates and upstream. Both at the inner and outer targets in the strike point vicinity, the Ne ion concentration is lower in the divertor than that at the omp.

Analysis of these simulations shows that the $\mathrm{E} \times \mathrm{B}$ drift and diffusive radial flows in the divertor region are comparable, similar to that found in the analogous modelling of ASDEX Upgrade [12]. The $\mathrm{D}$ ionization rate, integrated over the volume of each divertor is $3-5 \times 10^{24}$ particles $/ \mathrm{s}$. These ions are mostly transported poloidally by the parallel flows. Integrated along the target, the parallel flows are an order of magnitude larger than both the radial diffusive and drift flows, poloidally integrated along the divertor regions from the X-point to the target. The radial flows thus make only a modest contribution to the particle balance equation, which is dominated by the parallel flows and the ionization/recombination sources. It should be noted, however, that the parallel flows are arise partially as a result of closure of the drift flows. The poloidal $\mathrm{E} \times \mathrm{B}$ drifts are also slow in the divertor with respect to the poloidal projection of the parallel velocity - less than $10 \%$ - so their effect is again rather weak.

The low impact of drifts on the main ions particle balance is specific to this ITER simulation for both Cases \#1b and \#2b and differs from simulations of medium size tokamaks, such as ASDEXUpgrade [12] or DIII-D [24], where the main ion $\mathrm{E} \times \mathrm{B}$ drift transport from the outer to the inner divertor integrated over the PFR under semi-detached or attached conditions is comparable to the integral ionization source in the outer divertor volume. A qualitative treatment of the relative importance of the drifts for machines of different scale is given in [25], where SOLPS-ITER modeling results for ITER and ASDEX Upgrade with drifts activated are compared under conditions of partial detachment. 
At high $\mathrm{p}_{\mathrm{n}}$, therefore, the drift effect is much less significant in terms of target power loading on ITER in comparison with smaller devices.

Under lower throughput conditions (lower $\mathrm{p}_{\mathrm{n}}$, Case \#1) the effect of drifts on the target power loading in ITER is significant, Fig. $6(\mathrm{~g}, \mathrm{~h})$. The main factor responsible for this difference is the $\mathrm{Ne}$ redistribution when drifts are activated. The drifts tend to increase $\mathrm{n}_{\mathrm{Ne}}$ in the inner divertor region and decrease it in the outer, leading to a pronounced local minimum of $\mathrm{n}_{\mathrm{Ne}}$ in the outer divertor Fig.6(e,f). The main consequence is a strong rise in $T_{e}$ at the outer and a decrease at the inner, Fig. 6(c,d). In turn, this drives the outer target in to a less detached state, yielding a considerable rise of the peak power load to the divertor target in the presence of drifts. This effect of the Ne redistribution is very clearly reflected in the total radiation from the inner and outer divertor regions listed in Table 1. For Case \#1, the outer divertor radiation without drifts is provided mostly by the partially ionized impurities. This contribution decreases by about a factor 5 when the drifts are switched on.

For Case 2 at higher $\mathrm{p}_{\mathrm{n}}$, Ne redistribution from the outer to inner divertor also occurs, but does not lead to a change of the outer divertor regime, which remains partially detached, leading only to a moderate change in the outer target power load when drifts are activated.

\subsection{Neon distribution}

While the redistribution of impurities plays an important role in the divertor target power asymmetry and radiation, the poloidal distribution of $\mathrm{Ne}$ in the upstream SOL is of interest from the point of view of both radiation and core plasma accumulation. To obtain a qualitative picture of the impurity distribution, it is instructive to analyze the impurity density and the parallel forces summed over all the ionized states of impurity. The parallel velocity is taken as a density-weighted average over all $\mathrm{Ne}$ charge states, in the same way as in [26].

The poloidal density distributions of $\mathrm{D}$ and Ne ions in the near and far SOL regions are shown in Fig. 7. The observed, more pronounced impact of drifts on Ne than on the fuel ions can be explained by the different transport properties of the main plasma ions and impurities in the SOL and the divertor. The fuel ion distribution along the field lines is determined by the pressure distribution. Variation of pressure is weak above the region of ionization. The significant change in the main ion pressure distribution in the divertor above the ionization regions can be caused by ion-neutral friction, by radial $\mathrm{E} \times \mathrm{B}$ drift flows with divergences comparable to the ionization sources, or by poloidal $\mathrm{E} \times \mathrm{B}$ drift velocities comparable to the poloidal sound speed [27]. For ITER, the drift velocities are insufficient to change the pressure distribution. Therefore drifts have only a modest impact on the main ionsdensity distribution. Generally, the poloidal distribution of fuel ion density in the SOL is flat, cf. Fig.7(a,b), with the poloidal profile in the divertor region compensating the temperature drop towards the targets and the pressure drop below the ionization regions.

In contrast, the Ne distribution is more sensitive to the changes of the plasma parameters at the different fuel throughputs. The Ne parallel velocity is determined by parallel force balance [25, 26, 28 ], with the main contributors being the thermal force and friction with the $\mathrm{D}$ ions. The force balance demonstrating these main contributions is shown in Fig. 8 for a flux tube $7 \mathrm{~mm}$ from the separatrix at the omp as an example. For other flux tubes in the SOL this balance is qualitatively similar. The force balance determines the difference between the parallel velocities of the main and impurity ions, with minor contributions from the $\mathrm{Ne}$ pressure and the electric force. The parallel diffusion of $\mathrm{Ne}$ with respect to the main ions, proportional to the Ne pressure gradient contribution to the parallel force 
balance, is not significant. The poloidal and radial components of the $\mathrm{E} \times \mathrm{B}$ drift and the Ne parallel velocity are almost independent of the $\mathrm{Ne}$ density gradient, while the $\nabla \mathrm{B}$ drift contribution to the $\mathrm{Ne}$ particle balance is low. So the Ne density distribution should adjust itself to make the divergence of the parallel and drift flows equal to the $\mathrm{Ne}$ ionization source, or to compensate it by anomalous diffusive flow. This allows for steep impurity density gradients, necessary to drive anomalous diffusive flows and very sensitive to the details of the ExB drifts (common for all charged particles), the main ion parallel velocity profile and the main ion temperature gradient. Moderate changes of the velocity distribution due to the drifts can therefore affect the poloidal $\mathrm{Ne}$ density profile and its distribution near the targets, cf. Fig.7(c,d). The impact on the main ions is less pronounced, cf. Fig.7(a,b).

The maximum of the Ne density distribution in the upstream SOL (Fig.7(c,d)), can be explained by the effect of the thermal force. In the near SOL, both the D and Ne parallel velocities are directed mainly towards the top of the main chamber, Figs. 9, 10(a,c). This feature of the D velocity in ITER modeling is known from the SOLPS4.3 results database [16], obtained without drifts. The parallel flow directed from the region of intense ionization near the divertor targets towards the top of the tokamak arises in the near SOL. This flow, changing direction near the top of the machine, is closed by radial diffusion upstream and return parallel flows towards the divertor in the far SOL. Pfirsch-Schlüter main ion flows compensating the $\nabla \mathrm{B}$ drift enhance the near SOL parallel flows in the upstream direction, but their influence is moderate. To see this, the parallel velocities of the main ions in the near SOL with and without drifts are compared in Fig. 10(a). For Case \#2, in which the divertor regime did not change when drifts are turned on, the change in the parallel velocity can be attributed to these PfirschSchlüter flows. The thermal force increases the Ne parallel velocity directed upstream in the X-point vicinity where the poloidal gradient of the ion temperature is large, with respect to the parallel velocity of the main ions, see Figs. 10(a) and 10(c). As a result, the Ne poloidal density has a minimum at this poloidal position, providing a slowly changing parallel flow, proportional to the product of the velocity and density. In addition, the thermal force shifts the stagnation point of the Ne velocity with respect to that of the main ions towards the poloidal maximum of the temperatures, located at the omp.

The Ne poloidal velocity, determined by the main plasma parameters, changes sign somewhat above the omp, see Fig.10(e). This distribution of velocity leads to Ne accumulation in the vicinity of the poloidal velocity stagnation point. The local maximum of Ne density in the near SOL in turn leads to an increase of the radial diffusion to the far SOL, where the Ne ions are carried by the main ion flow towards the divertors. As a consequence, the direction of the poloidal velocity of the main ions in the far SOL region, where $\mathrm{Ne}$ arrives due to diffusion, is responsible for the subsequent transport of impurity either to the inner or outer divertor.

An interesting feature of the Ne density profile at the divertor targets is a local minimum in the near SOL, visible both with and without drifts in the outer divertor, see Fig. 6(f). This can be explained by the effect of finite ionization potential [26]. The latter from neutral $\mathrm{Ne}$ to $\mathrm{Ne}^{+}$is larger than the value from neutral $\mathrm{D}$ to $\mathrm{D}^{+}$. On near SOL flux surfaces, where the $\mathrm{D}$ flow is directed upstream (Fig. 9), the D poloidal flow has a stagnation point. Below this point, all the D ionized on these flux tubes moves towards the target, while the relatively small fraction of the D neutrals ionized above this point move towards the main chamber. Neon atoms can reach this stagnation point with a higher probability than $\mathrm{D}$ atoms due to their larger ionization potential and therefore smaller ionization cross-section for typical divertor temperatures. As a result, the Ne ions are dragged towards the main chamber SOL 
with higher probability so that on the flux tubes where the flow is directed upstream, $\mathrm{n}_{\mathrm{Ne}}$ near the target decreases.

In the high $\mathrm{p}_{\mathrm{n}}$ Case \#2, the main plasma parameters - power load to the targets, $\mathrm{n}_{\mathrm{e}}, \mathrm{T}_{\mathrm{e}}$ in the divertor and at the omp - do not change significantly when drifts are activated, Figs.5-6. It can therefore be expected that, in general, the plasma background including main plasma parallel velocity for impurities does not change significantly. The distribution of the main and impurity ion parallel velocities is thus only slightly affected by drifts: the difference between the parallel velocities in the SOL is $<10 \mathrm{~km} / \mathrm{s}$, less than $2 \mathrm{~km} / \mathrm{s}$ in their poloidal projections and about $200 \mathrm{~m} / \mathrm{s}$ in the poloidal $\mathrm{E} \times \mathrm{B}$ drift velocity. However, due to the sensitivity of $\mathrm{Ne}$ to the details of the main ion velocity and poloidal $T_{i}$ variation, these differences are sufficient to change the Ne poloidal distribution in the SOL and divertor plasma: the difference in the SOL poloidally averaged separatrix concentration for the same Ne seeding rate is $60 \%$ (Table 1) and about $30 \%$ in the divertor Ne densities, see Fig. 6. It should be noted that although the drifts do lead to the accumulation of Ne upstream and in the inner divertor, and to a $\mathrm{Ne}$ density decrease in the outer divertor, both divertors remain in the partially detached regime when drifts are switched on. Figure 11 clearly illustrates that the fraction of energy flow lost by the plasma on its way towards the outer divertor target does not change significantly when drifts are activated. The Ne concentration in the outer divertor is still sufficient for re-radiation of a considerable part of this energy, Table 1, ensuring that the divertor power load in-out asymmetry does not change considerably.

At low $\mathrm{p}_{\mathrm{n}}$, the redistribution of Ne due to the drifts, seen already for the detached state, leads to non-linear consequences. As a result, the outer target re-attaches and the loss of plasma energy in the outer divertor decreases, as seen in Fig. 11. The plasma temperature in the outer divertor rises, while $n_{D}$ decreases, Fig.6. An additional flow of ions through the SOL from the outer to the inner divertor appears, Fig.12. This flow partially compensates the neutral flow through the sub-divertor structures [16,29], from the inner to the outer divertor, which arises due to neutral pressure asymmetry between the divertors. In contrast to the SOLPS4.3 simulations, in SOLPS-ITER modeling the flow from the sub-divertor structures towards the outer divertor in both Cases \#1a and \#2a in the absence of drifts is small compared to the deuterium puffing flow. This difference in modeling results is associated with the more symmetric divertor conditions obtained in SOLPS-ITER without drifts (see Section 2.5 for analysis of divertor asymmetry in SOLPS-ITER and SOLPS4.3 modeling). In both

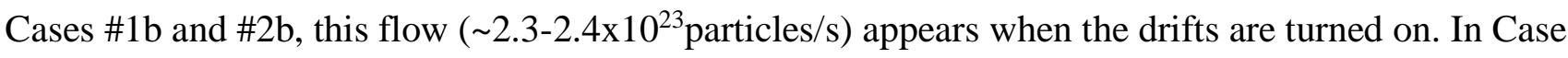
\#2b, this flow is compensated by the parallel and ExB drift flows through the PFR, while in Case \#1b it is partially compensated by flow through the PFR and partially through SOL. The change in the parallel and poloidal velocities in the SOL as the drifts are turned on is therefore considerable for Case \#1, for both D and Ne ions, cf. Fig. 10. Impurity leakage from the outer divertor increases, leading to a peak in-out asymmetry in the target Ne densities increasing from 1:2 to $6: 1$.

\subsection{Global scalings; comparison with SOLPS4.3 results}

As mentioned in Section 2.1, probably the most important feature of the ITER SOLPS simulation database is the operating domain of $\mathrm{q}_{\mathrm{pk}}$ and $\mathrm{p}_{\mathrm{n}}$ for the divertor. The former must be within steady state power handling limit of the $\mathrm{W}$ monoblocks constituting the high heat flux regions of the divertor targets, whilst the latter must be sufficient to ensure adequate He exhaust in burning plasma regimes. This $q_{p k}, p_{n}$ operational space was first established with SOLPS-4.3 [15] and has recently been the 
subject of further detailed examination [16]. It has been the basis upon which the first ITER W divertor design was consolidated after many years of simulation studies which focused on the initial ITER strategy of beginning operation with carbon material in the strike point regions. In [16], a subset of the first results for the dependence of $\mathrm{q}_{\mathrm{pk}}$ on $\mathrm{p}_{\mathrm{n}}$ obtained in the study described here were included for comparison with the SOLPS-4.3 database (which does not include drifts). Here, the larger SOLPSITER drift activated runs are now presented, along with a more detailed discussion of the reasons for the differences found between the two codes at given operating points.

The SOLPS-4.3 Ne-seeded database consists of a series of scans of fuel and impurity throughput such that sets of curves are available in which $\mathrm{p}_{\mathrm{n}}$ is scanned from low (attached) to high (detached) conditions at approximately constant $\mathrm{c}_{\mathrm{Ne}}$, with the $\mathrm{Ne}$ concentration defined by the poloidally averaged main chamber near SOL metric defined in Section 2.1. Reproducing the full SOLPS-4.3 scan, which contains more than 100 validated entries, with SOLPS-ITER including drifts is an ongoing task and is challenging, even with the new speed-up schemes described in [11]. However, a full throughput scan across the SOLPS-4.3 range of $\mathrm{p}_{\mathrm{n}}$ does now exist for an approximately constant $\mathrm{c}_{\mathrm{Ne}}$ in the range $0.35-2 \%$. The results in the $\mathrm{p}_{\mathrm{n}} \mathrm{vs}$. $\mathrm{q}_{\mathrm{pk}}$ domain are shown in Fig. 13. They show the same trend as found with SOLPS-4.3 without drifts but give considerably higher peak power loading at the outer target for $\mathrm{p}_{\mathrm{n}}$ lower than about $7 \mathrm{~Pa}$, corresponding to high recycling and attached conditions and due to the redistribution of $\mathrm{Ne}$ driven by the drifts described in the previous section. For higher $\mathrm{p}_{\mathrm{n}}=8-10 \mathrm{~Pa}$, the same outer target semi-detached conditions and approximately similar in-outer peak power load asymmetries (see also Fig. 14) found with SOLPS-4.3 are recovered with SOLPS-ITER with or without drifts activated.

The divertor asymmetry in SOLPS-ITER modeling increases when the drifts are turned on compared to the case when they are deactivated, Fig. 14, for $\mathrm{p}_{\mathrm{n}}<7 \mathrm{~Pa}$ and outer divertor $\mathrm{q}_{\mathrm{pk}}>8 \mathrm{MW} / \mathrm{m}^{2}$. While in the SOLPS-ITER scan the inner divertor in the absence of drifts is even slightly hotter than the outer, and the asymmetry level does not depend on $\mathrm{p}_{\mathrm{n}}$ or $\mathrm{q}_{\mathrm{pk}}$ at the outer target (Fig 14), in the SOLPS4.3 scans the outer divertor is hotter for $\mathrm{p}_{\mathrm{n}}<7 \mathrm{~Pa}$ and the asymmetry increases with decreasing of $\mathrm{p}_{\mathrm{n}}$.

The cause of the significant discrepancy in the asymmetry between the different scans (SOLPS4.3 without drifts and SOLPS-ITER with and without drifts) has been studied using a technique [9] in which SOLPS-ITER is first run in a way in which code flags are set to mimic the earlier SOLPS-4.3 simulations. At low $\mathrm{p}_{\mathrm{n}} \sim 3 \mathrm{~Pa}$, SOLPS-ITER run in this way finds a considerable out-in $\mathrm{q}_{\mathrm{pk}}$ asymmetry $(\sim 2: 1)$, very close to the value computed with SOLPS -4.3 for approximately similar $\mathrm{c}_{\mathrm{Ne}}$ (note that the $\mathrm{q}_{\mathrm{pk}}$ asymmetry is roughly independent of $\mathrm{c}_{\mathrm{Ne}}$ at low $\mathrm{p}_{\mathrm{n}}$ in the SOLPS-4.3 database [16]). The SOLPSITER simulations are then transitioned by progressively changing the run parameters to activate the recent improvements of the code. Among these parameters are: inclusion of metric coefficients accurately describing toroidicity of the system in the parallel momentum balance; radial currents due to classical and anomalous viscosity; changes of the numerical scheme from a 5-point to the 9-point stencil in particle and heat balance, necessary to include drifts; restriction of the minimum ion density from $10^{4} \mathrm{~m}^{-3}$ to $10^{8} \mathrm{~m}^{-3}$ for each ion species and charge, necessary for numerical reasons when drifts are activated; flux limiting scheme for the ion parallel heat conductivity inside the separatrix, necessary to produce the correct solution for neoclassical ion heat transport if drifts are switched on [3]; scheme flux limiting scheme for the ion parallel heat conductivity outside the separatrix; accurate expression for the parallel viscosity and the term associated with the parallel heat flow on the RHS of the parallel momentum balance, required to yield the correct solution for the neoclassical electric field if drifts are 
activated [3]; changes in boundary conditions necessary to include drifts. As shown by the blue squares in Fig. 14, this yields a nearly symmetric in-out peak power load when drifts are deactivated, thus directly contradicting the SOLPS-4.3 result. By then progressively activating the various changes to the physics model in SOLPS-ITER which have been made since the original SOLPS-4.3 version was generated, it is possible to identify the critical parameter responsible for driving the solution to a more symmetric load (in the absence of drifts).

Following this procedure, it was found, in fact, that the critical change was the enforcement of an ion heat flux limiter outside the separatrix in SOLPS-ITER, not active in SOLPS-4.3. This flux limiter is a restriction on the ion heat conductive energy flow to prevent it exceeding the convective ion energy transport with some fraction of the sound speed velocity [3]. Technically it is applied as a transformation of the parallel heat conductivity $\kappa_{i \|}$ :

$$
\tilde{\kappa}_{i \|}=\kappa_{i \|} \cdot\left(1+\frac{\kappa_{i \|} b_{x}^{2}}{c_{i}^{\lim } b_{x} T_{i} n \sqrt{T_{i} / m_{i}}} \frac{\partial T_{i}}{h_{x} \partial x}\right)^{-1}
$$

where the variables correspond to the definitions in [3]. Historically in SOLPS4.3, due to convergence reasons, the coefficient $c_{i}^{\lim }$ was chosen equal to $10^{5}$, effectively rendering flux limiting inactive. In the SOLPS-ITER code configuration, the more physically justified parameter $c_{i}^{\lim }=0.6$ [30] is applied. Turning on only this parameter with all others unchanged leads to a transition to the symmetric $\mathrm{q}_{\mathrm{pk}}$ solutions. It is, however, important to note that the transition from a symmetric to an asymmetric SOLPS-ITER solution (in terms of peak power load) simply by turning off ion heat flux limiting is not always possible. At low $\mathrm{p}_{\mathrm{n}}$ (and for the $\mathrm{c}_{\mathrm{Ne}} \sim 1 \%$ case considered so far), turning off ion flux limiting in SOLPS-ITER leads to asymmetric divertor power loads if the initial state was asymmetric (taken from the SOLPS-4.3 mimicking case) and to symmetric power loads for a SOLPS-ITER symmetric initial state. For higher $\mathrm{p}_{\mathrm{n}}>5 \mathrm{~Pa}$, the solution is unique, the power load is comparatively small (about $\lesssim 6 \mathrm{MW} / \mathrm{m}^{2}$ ) and symmetric, and the solution is insensitive to the ion heat flux limits.

Further analysis of the SOLPS-ITER simulations reveals similar impurity behaviour to that discussed in the previous section: asymmetric power loading without drifts when the ion flux limiting is turned on is associated with impurity accumulation in the inner divertor and attached outer target conditions with low impurity concentrations, while symmetric solutions correspond to a semi-detached outer divertor with a higher Ne density than in the inner divertor.

Asymmetric solutions lead to net fuel flow from the outer to the inner target through the SOL, participating in the redistribution of $\mathrm{Ne}$ in the divertors, and resembling the flow through the SOL at low $\mathrm{p}_{\mathrm{n}}$ with drifts on. Such a flow was also reported in SOLPS4.3 modelling [29]. An important finding from the study reported here is that when the divertor neutral pressure is low (below about $6 \mathrm{~Pa}$ ), the SOLPS-ITER solution is sensitive to the details of the modelling set-up as a consequence of the sensitivity of the impurity distribution to these details. On one possible solution branch, the impurities tend to accumulate in the inner divertor, while on the other a more balanced distribution is found. Symmetric solutions can be rendered asymmetric by removing the flux limit for ion heat flow, even though the poloidal distribution of the ion temperature is hardly affected. Likewise, a symmetric solution can be made asymmetric by activating drifts, even though this has no significant effect on D 
flows and upstream plasma parameters. The impurity distribution calculations in all cases require a numerical approach - simple analytical arguments cannot provide even a qualitative picture of the impurity redistribution. Regimes with $\mathrm{p}_{\mathrm{n}}$ less than about $7 \mathrm{~Pa}$, within the current operating window resulting from the code inputs in this particular study (e.g. for givenengineering pumping speed) are can lead to rather large excursions in peak power loading compared to the previous drift-free simulations because small changes in the background plasma can lead to non-linear consequences as a result of subtle changes to the redistribution of impurities driving outer divertor reattachment.

\section{Conclusions}

Modeling of ITER neon-seeded, H-mode baseline inductive burning plasmas (power into the scrapeoff layer of $100 \mathrm{MW}$ ) with the SOLPS-ITER code shows that under conditions of partial divertor detachment, corresponding to sub-divertor neutral pressures $\sim 10 \mathrm{~Pa}$, the inclusion of fluid drifts in the simulations does not significantly modify the distributions of the density and temperature of the main ions, nor the power loading of the divertors, compared to the case without drifts. At lower pressures, when the divertor approaches the high recycling then attached states, the drift effect is more dramatic. Redistribution of the neon impurity can lead to a considerable increase of the outer divertor plasma temperature, the peak power load and the out-in divertor power load asymmetry.

This is a key finding of the unique new simulations presented in this paper: if the ITER divertor does find itself in an operational state in which neutral pressure is low (e.g. in a divertor reattachment event, for example due to loss of impurity seeding), the outer target power load could rise very rapidly to values considerably higher than found in the previous SOLPS-4.3 simulation database which did not include fluid drifts in the model. This would place even tighter constraints on detachment control, given that the 2D simulation results here do not account for divertor component shaping, which, on ITER, will increase heat loads by $\sim 50 \%$ in the case of hot attached divertor plasmas in which the load is dominated by the thermal plasma and ion recombination at the plates [16].

Analysis of the radial and poloidal profiles of the electric field in the edge transport barrier region, SOL and divertor demonstrates that the behaviour of the ITER discharges chosen for this modeling is qualitatively similar to that observed in the devices such as ASDEX-Upgrade in partially detached regimes. In particular, the radial electric field in the transport barrier is of the order of the neoclassically expected value. Outside the separatrix, the poloidal electric field balances the electron pressure gradient and the thermal force except in the private flux region, where the electric conductivity is low and the potential is determined by the parallel current.

Neon density peaking is predicted in the near SOL, due to the thermal force and frictional drag with the main ions. The Ne distribution is more sensitive to the drifts than the main ions, with the consequence that drifts lead to a considerable decrease of the Ne concentration in the outer and increase in the inner divertor. As stated earlier, this impurity redistribution as a result of the drifts is what drives the increasing out-in asymmetry at low neutral pressure. Impurity retention in the divertor regions is reduced with drifts activated compared to the no drift case.

Until the new SOLPS-ITER drift simulations reported here became available, the ITER divertor physics basis was entirely founded on an extensive SOLPS-4.3 simulation database [15,16], where drifts could not by default be included (drift terms are not present in the B2 fluid code used by SOLPS-4.3). It was thus important to compare the new simulation results with equivalent SOLPS-4.3 cases. The major differences are found at low divertor neutral pressure, where SOLPS-ITER without 
drifts finds a lower out-in peak power load asymmetry. This was traced to the impact of ion heat flux limiting, which is included in the SOLPS-ITER model, but is absent in SOLPS-4.3. Switching it off in the new simulations brings the SOLPS-ITER and SOLPS-4.3 code results into agreement. At higher pressures, the effect is much reduced and the two codes are in good agreement in absolute magnitude of the peak heat loads at both targets for approximately similar Ne impurity concentration. Thus, at low neutral pressure, the ion heat flux limiting in SOLPS-ITER pushes the out-in power load asymmetry down, but the drifts increase it even more so that the overall effect is an increase in the asymmetry in comparison with the previous SOLPS-4.3 results.

This paper provides a comforting demonstration that the inclusion of drift effects in ITER divertor plasma simulations does not significantly modify the partially detached solutions found previously without drifts at high sub-divertor neutral pressure and input power consistent with burning plasma operation. Operationally important differences appear at lower neutral pressure due to the drift effect on the distribution of seeded impurity. As a result, a more complete assessment is now required, in which the existing full SOLPS-4.3 database is reproduced with SOLPS-ITER, including drifts. Such studies must extend the range of impurity concentration, examine the influence of input power and investigate the differences across the full operational range of using nitrogen seeding as an alternative to neon.

\section{Acknowledgements}

This work was supported by the Russian Science Foundation, grant no. 17-12-01020. Numerical calculations were performed at the Polytechnic Supercomputer Center at Peter the Great St. Petersburg Polytechnic University.

The views and opinions expressed herein do not necessarily reflect those of the ITER Organization.

\section{References}

[1] Kukushkin A.S., Pacher H.D., Kotov V., Pacher G.W., Reiter D. 2011 Fusion Eng. Des. 86 28652873

[2] Kotov V., Reiter D., Pitts R. A., Jachmich S., Huber A., Coster D. P. and JET-EFDA contributors 2008 Plasma Phys. Control. Fusion 50105012

[3] Rozhansky V., Kaveeva E., Molchanov P., Veselova I., Voskoboynikov S., Coster D., Counsell G., Kirk A., Lisgo S. ASDEX-Upgrade Team and MAST Team 2009 Nucl. Fusion 49025007

[4] Reimold F., Wischmeier M., Potzel S., Guimarais L., Reiter D., Bernert M., Dunne M., Lunt T., the ASDEX Upgrade Team, the EURO fusion MST1 Team 2017 Nucl. Materials and Energy 12193

[5] Chankin A. V., Campbell D. J., Clement S., Davies S. J., Horton L. D., Stamp M. and Stangeby P. C. 1996 Plasma Phys. Control. Fusion 381579

[6] Smick N., LaBombard B. and Hutchinson I.H. 2013 Nucl. Fusion 53023001

[7] Pitts R. A. et al 2005 J. of Nucl. Mater. 337-339 146-153 
[8] Bonnin X., Dekeyser W., Pitts R., Coster D., Voskoboynikov S. and Wiesen S. 2016 Plasma Fusion Res. 111403102

[9] Wiesen S. et al 2015 J. Nucl. Mater. 463480

[10] Vekshina E., Senichenkov I., Rozhansky V., Kaveeva E., Khromov N., Kurskiev G., Patrov M. and Globus-M team 2016 Plasma Phys. Control. Fusion 58085007.

[11] Kaveeva E., Rozhansky V., Senichenkov I., Veselova I., Voskoboynikov S., Sytova E.

Bonnin X., Coster D. 2018 Nucl. Fusion 58126018

[12] Senichenkov I., Kaveeva E., Rozhansky V., Sytova E., Veselova I., Voskoboynikov S., Coster D. 2015 Proc. 42 EPS Conf. on Controlled Fusion and Plasma Physics (Lisbon, Portugal) ECA Vol. 39E P5.191

[13] Dekeyser W., Bonnin X., Lisgo S.W., Pitts R.A., Brunner D., LaBombard B., Terry J.L. 2017 Nucl. Mater. Energy 12 899-907

[14] Meier E. T., Goldston R. J., Kaveeva E. G., Makowski M. A., Mordijck S., Rozhansky V. A., Senichenkov I. Yu. and Voskoboynikov S. P. 2016 Plasma Phys. Control. Fusion 58125012

[15] Pacher H.D., Kukushkin A.S., Pacher G.W., Kotov V., Pitts R.A., Reiter D. 2015 J. Nucl. Mater. $463591-595$

[16] R. A. Pitts et al 2019 Nucl. Mater. Energy 20100696

[17] Park J.-S. et al in preparation for Nucl Fusion

[18] Kukushkin A. S., Pacher H. D., Kotov V., Reiter D., Coster D. P., Pacher G. W. 2007 J. Nucl. Mater. 363-365 308-313

[19] Kukushkin A. S. et al. 2003 Nuclear Fusion 43716

[20] Pacher H. D. et al 2007 J. Nucl. Mater., 363-365 400-406

[21] Eich T. et al 2013 Nucl. Fusion 53093031

[22] Chang C.S. et al 2017 Nucl. Fusion 57116023

[23] Rozhansky V., Kaveeva E., Veselova I., Voskoboynikov S. and Coster D. 2016 Contrib. to Plasma Phys. 56 587-591

[24] Jarvinen A. E. et al 2018 Phys. Rev. Letters 121075001 
[25] Sytova E., Pitts R. A., Kaveeva E., Bonnin X., Coster D., Rozhansky V., Senichenkov I., Veselova I., Voskoboynikov S., Reimold F. 2019 Nucl. Materials and Energy 19 72-78

[26] Senichenkov I.Yu., Kaveeva E.G., Sytova E.A., Rozhansky V.A., Voskoboynikov S.P., Veselova I.Yu., Coster D.P., Bonnin X., Reimold F., and the ASDEX-Upgrade Team 2019 Plasma Phys. Control. Fusion 61045013

[27] Stangeby P. C. "The plasma boundary of magnetic fusion devices” 2000 IOP publishing Ltd.

[28] Sytova E., Senichenkov I., Kaveeva E., Rozhansky V., Veselova I., Voskoboynikov S., Coster D. 2016 Proc. 42 EPS Conf. on Controlled Fusion and Plasma Physics (Leuven, Belgium) ECA Vol. 40E $\mathrm{P} 1.054$

[29] Pshenov A. A., Kukushkin A. S., and Krasheninnikov S. I. 2017 Phys. Plasmas 2472508

[30] Igitkhanov Yu.L., Kukushkin A.S. and Runov A.M. 1994 Proc. 21st EPS Conf. on Controlled Fusion and Plasma Physics(Montpellier, France) ECA Vol. 18B 766-769. 


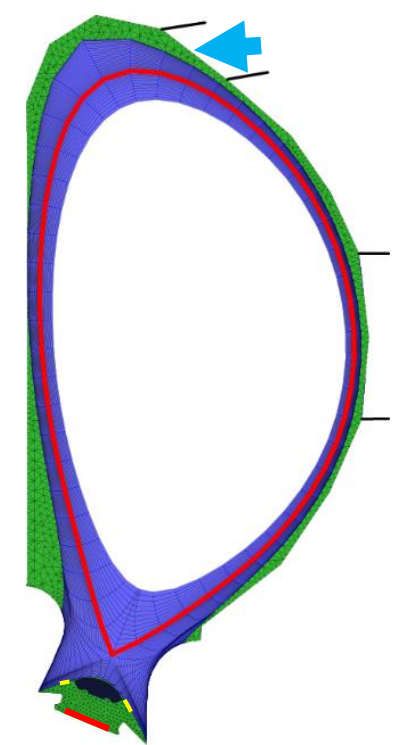

(a)

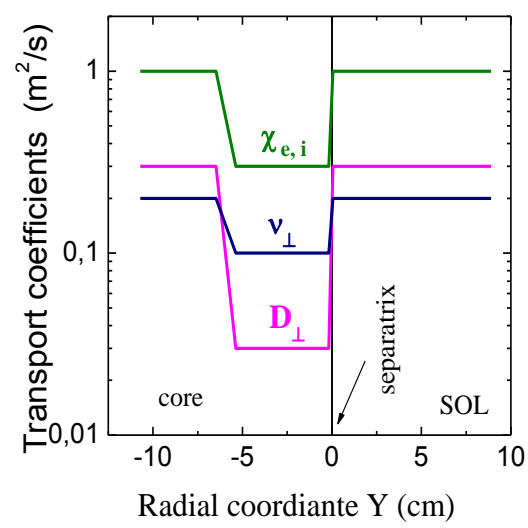

(b)

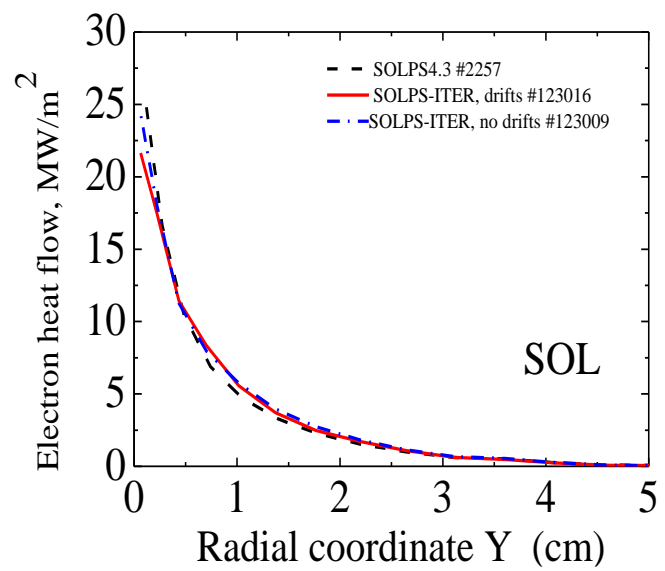

(c)

Fig.1.(a) Computational grid for the B2.5 fluid plasma solver (blue, quadrangles) and grid expansion for EIRENE (green, triangles). The main chamber D gas puffing location is marked by the blue arrow and the pumping surface under the dome with red line. The flux tube where the average $\mathrm{Ne}$ concentration, $\mathrm{c}_{\mathrm{Ne}}$ is calculated is shown in red with the region over which $\mathrm{p}_{\mathrm{n}}$ is computed shown in yellow (figure adapted from [16]). (b) Radial profiles of anomalous radial diffusion, viscosity and heat conductivity coefficients, showing clearly the H-mode pedestal region with reduced transport. (c) Parallel heat flow of electrons at the X-point level divided by flux tube cross-section at the target and plotted versus distance at the omp for cases with $\mathrm{p}_{\mathrm{n}}$ in the range $6.5-7.5 \mathrm{~Pa}$ and $\mathrm{q}_{\mathrm{pk}}=6-7.5 \mathrm{MW} / \mathrm{m}^{2}$ comparing SOLPS-ITER with and without drifts and a comparable SOLPS4.3 simulation. Numbers of calculations in the SOLPS-ITER database (Integrated Modelling Analysis Suite, IMAS database) are given in the plot.

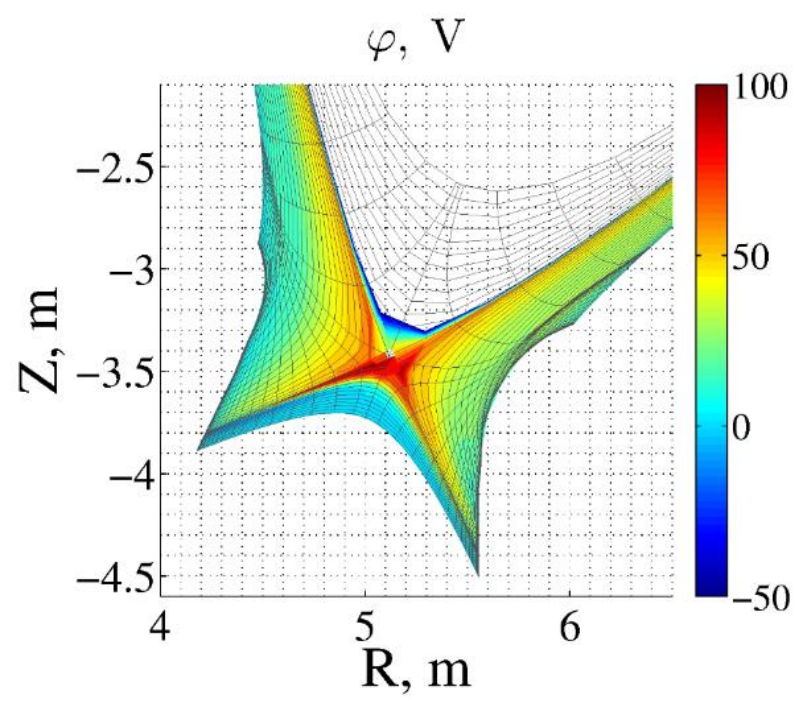

Fig. 2. Distribution of electrostatic potential in the divertor for Case \#2b, Table 1. The white regions inside the separatrix correspond to very negative potentials, down to $-3 \mathrm{kV}$, the second scenario. 


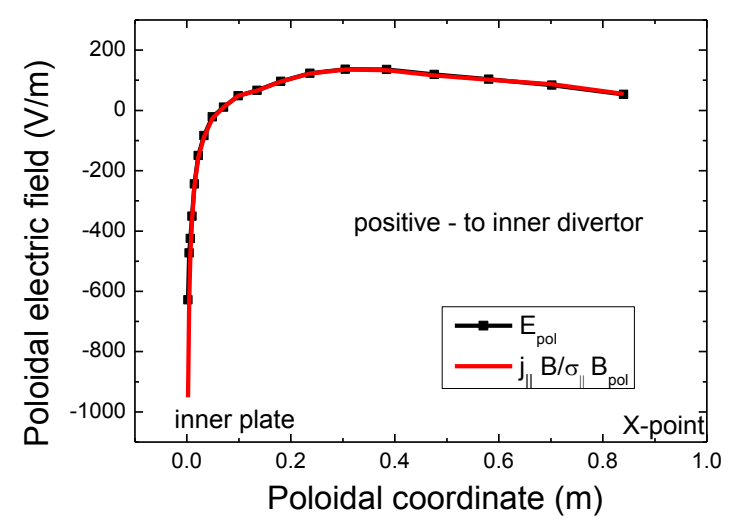

(a)

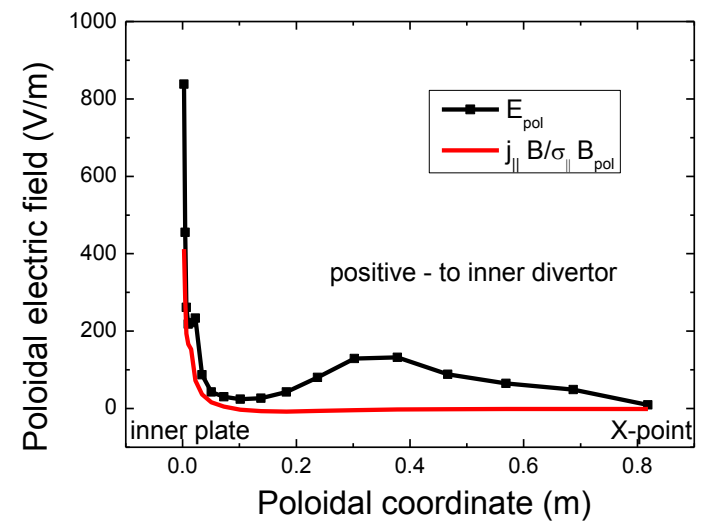

(b)

Fig. 3. Poloidal electric field profiles for Case \#2b and the associated parallel current (a) in the PFR, along the flux surface intersecting the inner target $11 \mathrm{~mm}$ from the strike point and (b) in the SOL on the flux surface intersecting the inner target $13 \mathrm{~mm}$ from the strike point $\left(\left(\mathrm{r}-\mathrm{r}_{\mathrm{sep}}\right)_{\mathrm{omp}}=2 \mathrm{~mm}\right)$.

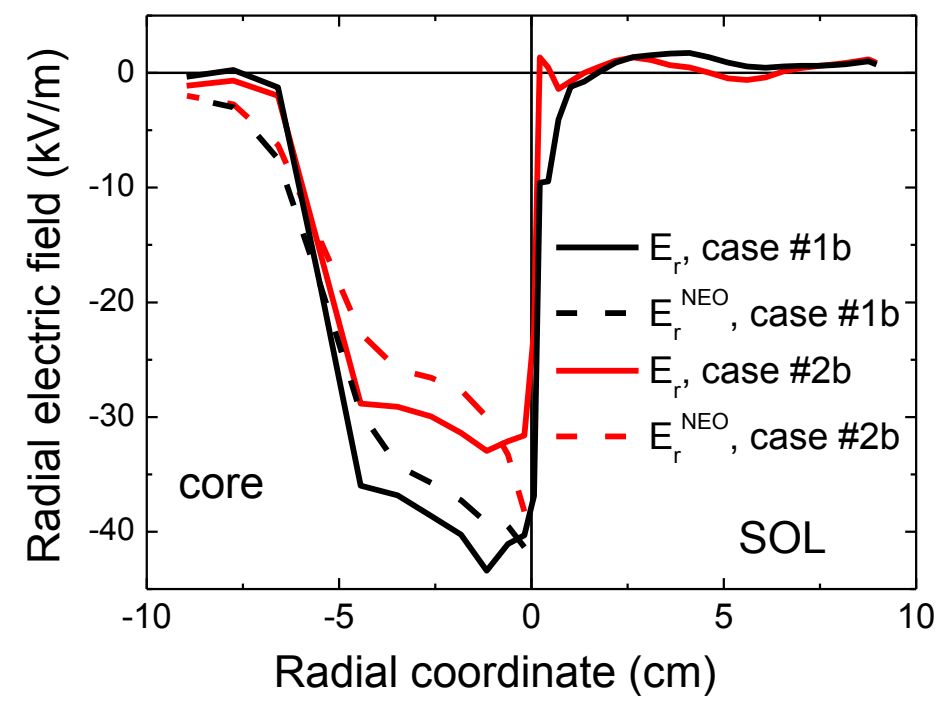

Fig. 4. Radial electric field profile at the omp for the low and high fuel throughput drift cases in Table 1. The vertical line marks the separatrix position. 


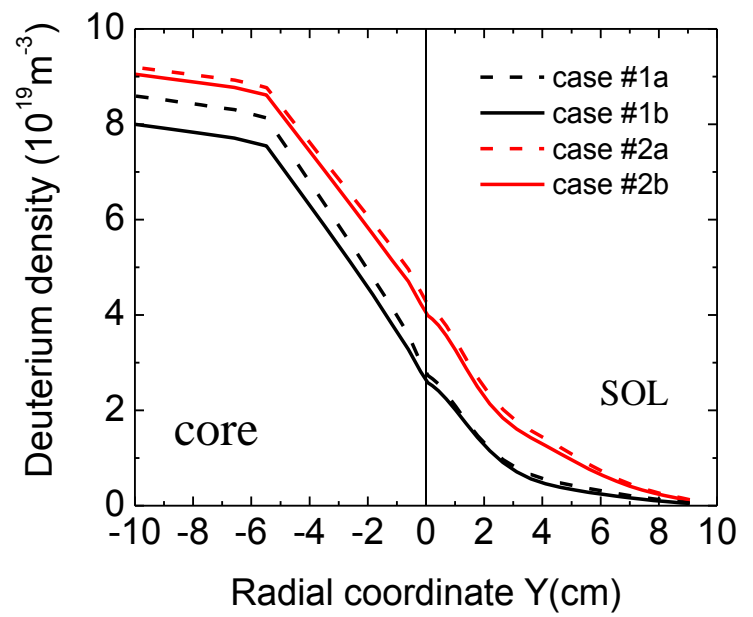

(a)

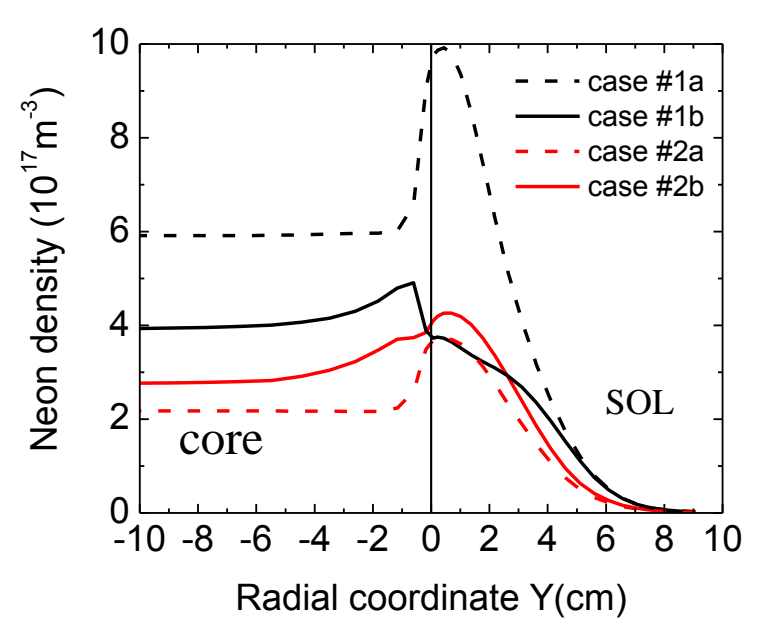

(c)

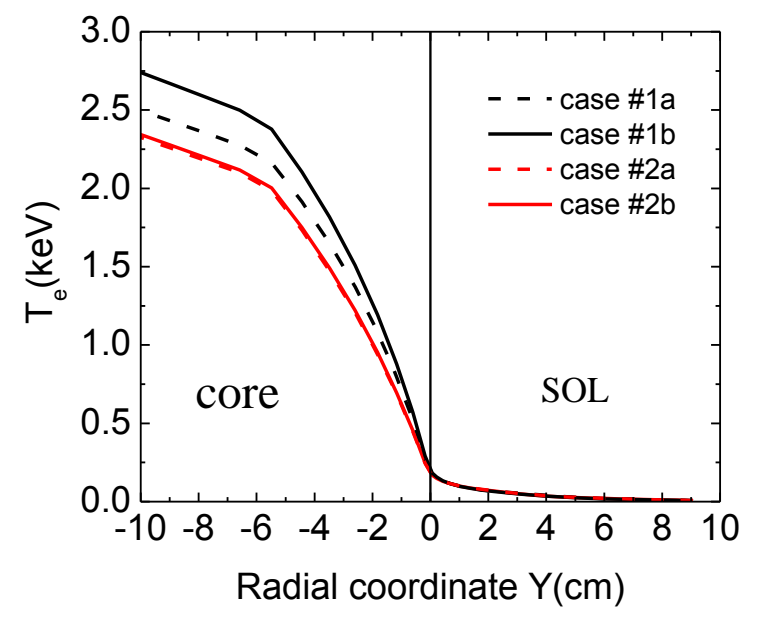

(b)

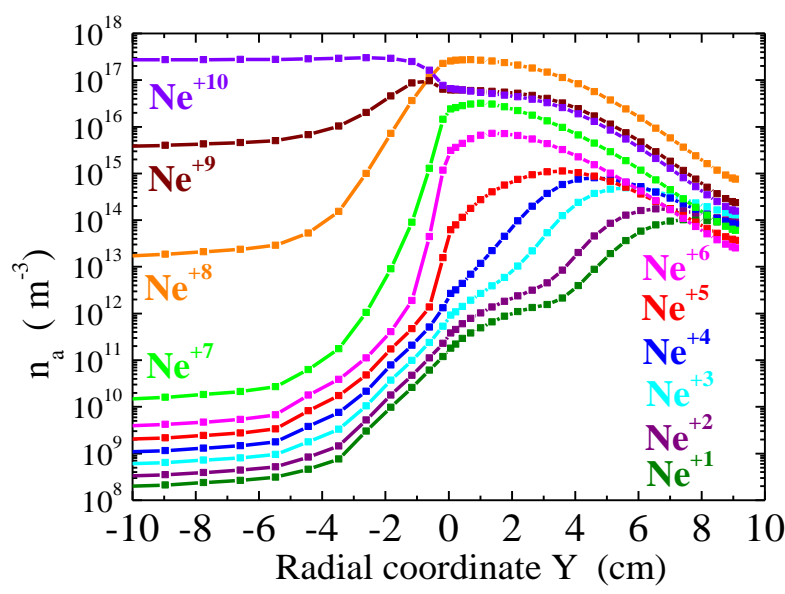

(d)

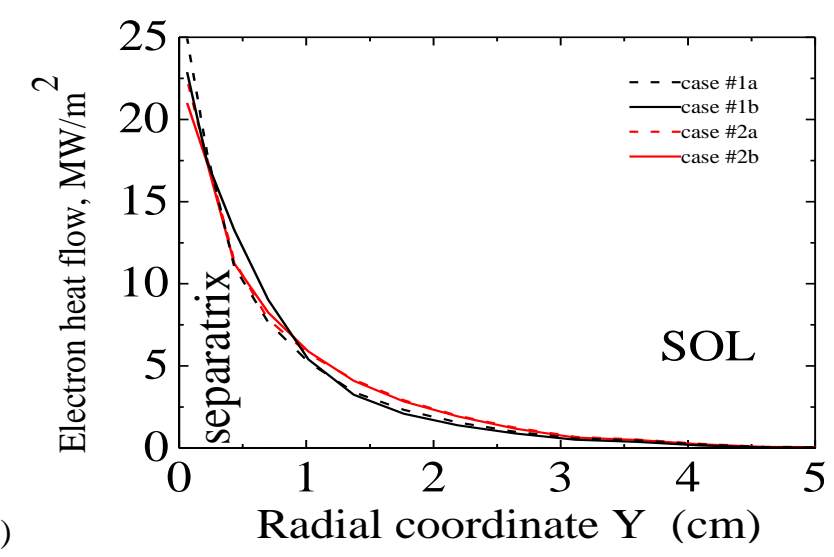

Fig. 5. Radial profiles at the omp of (a) $n_{D}$ (b) $T_{e}$ (c) $n_{N e}$ (summed over all charge states), for Case 1 (black) and Case \#2 (red) (d) densities of the different Ne charge states for Case \#2b (e) parallel heat flow of electrons at the X-point level divided by flux tube cross-section at the target and plotted versus distance at the omp. The vertical lines mark the separatrix position. 


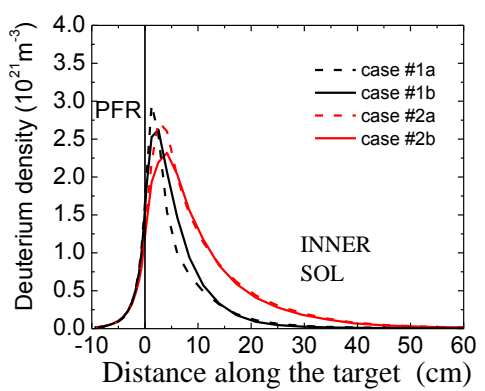

(a)

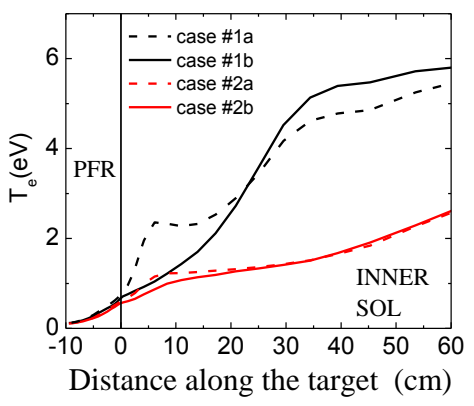

(c)

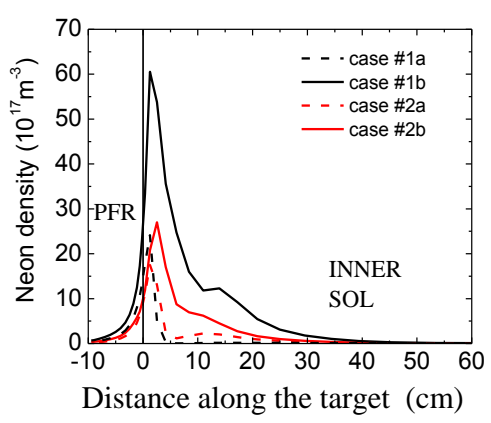

(e)

$(\mathrm{g})$
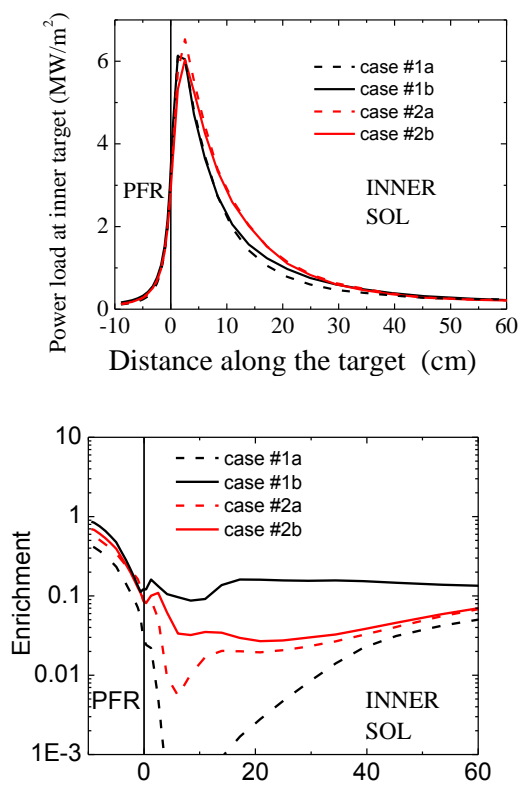

(i)
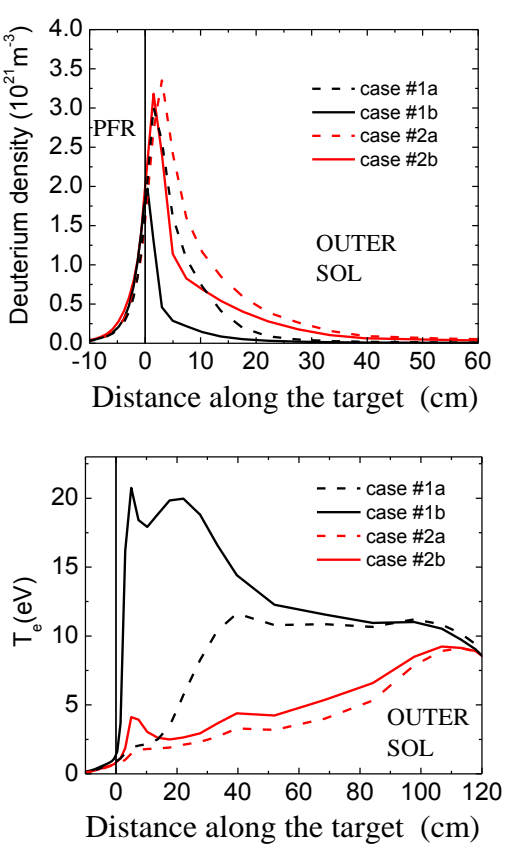

(d)

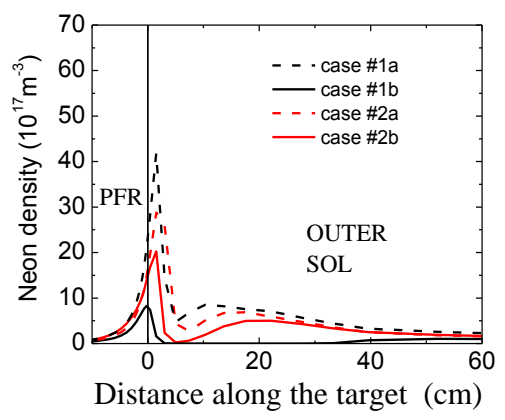

(h)
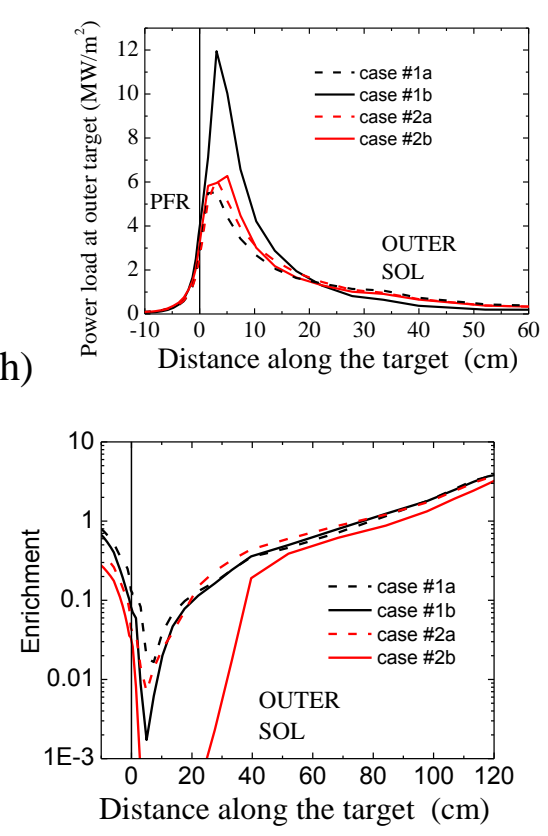

Fig.6. Radial profiles at the inner (left) and outer (right) divertor targets (Case 1 (black) and Case \#2 (red)). (a,b) $\mathrm{n}_{\mathrm{D}},(\mathrm{c}, \mathrm{d}) \mathrm{T}_{\mathrm{e}}$, (e,f) $\mathrm{n}_{\mathrm{Ne}}$ (summed over all charge states), (g,h) target power load, (i,j) enrichment calculated as $R=\frac{n_{N_{\_} d i v} \cdot n_{D_{-} o m p}}{n_{N_{-} o m p} \cdot n_{D_{-} d i v}}$. The vertical lines denote the strike point position. 


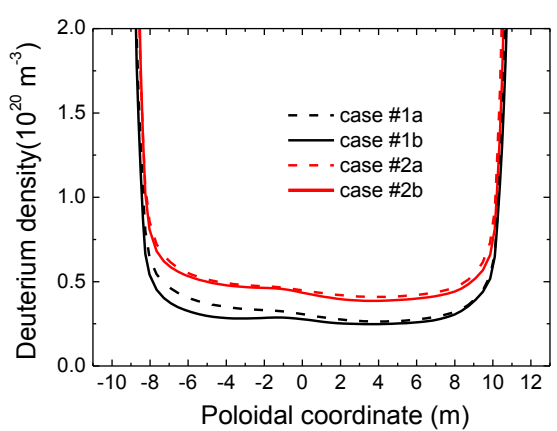

(a)

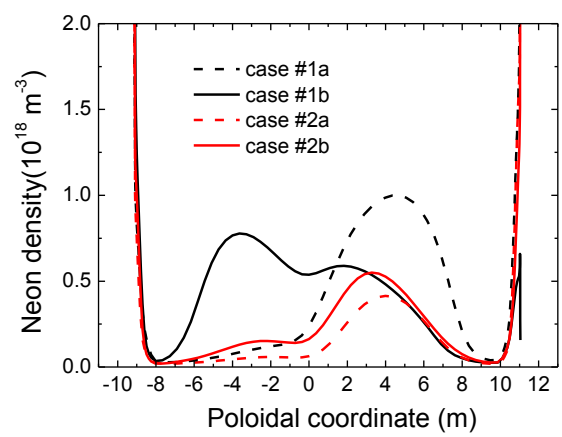

(c)

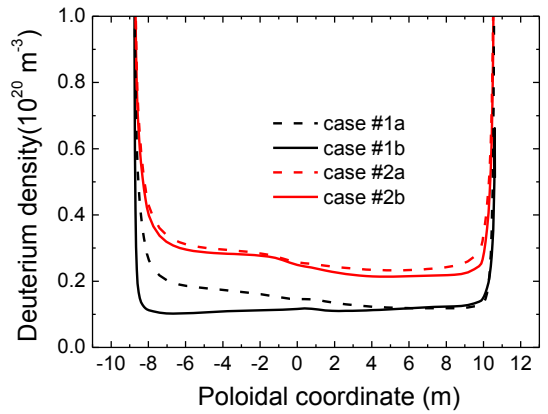

(b)

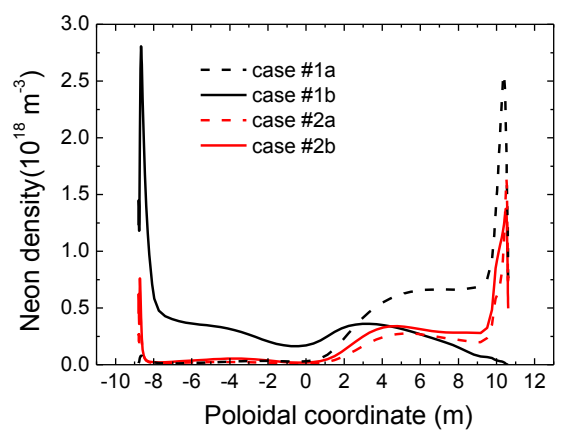

(d)

Fig.7. Poloidal distribution of (a) near SOL $n_{D}$ at $\left(r-r_{s e p}\right)_{o m p}=2 \mathrm{~mm}$, (b) far SOL $n_{D}$ at $\left(r-r_{s e p}\right)_{o m p}=22$ $\mathrm{mm}$, (c) near SOL $\mathrm{n}_{\mathrm{Ne}}$ at $\left(\mathrm{r}-\mathrm{r}_{\mathrm{sep}}\right)_{\mathrm{omp}}=2 \mathrm{~mm}$, (d) far SOL $\mathrm{n}_{\mathrm{Ne}}$ at $\left(\mathrm{r}-\mathrm{r}_{\mathrm{sep}}\right)_{\mathrm{omp}}=22 \mathrm{~mm}$. Case \#1 (black) and Case \#2 (red). Ne densities are the sum of all charge states. The X-point positions are $-8.3 \mathrm{~m}$ and $10.1 \mathrm{~m}$.

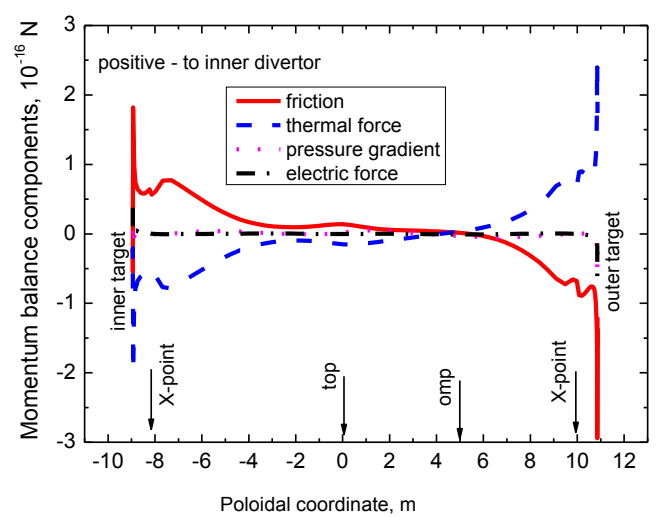

Fig.8. Parallel momentum balance per Ne ion (averaged overall ionized states) in the SOL at $\left(r-r_{\text {sep }}\right)=$ $7 \mathrm{~mm}$, case \#2b. 


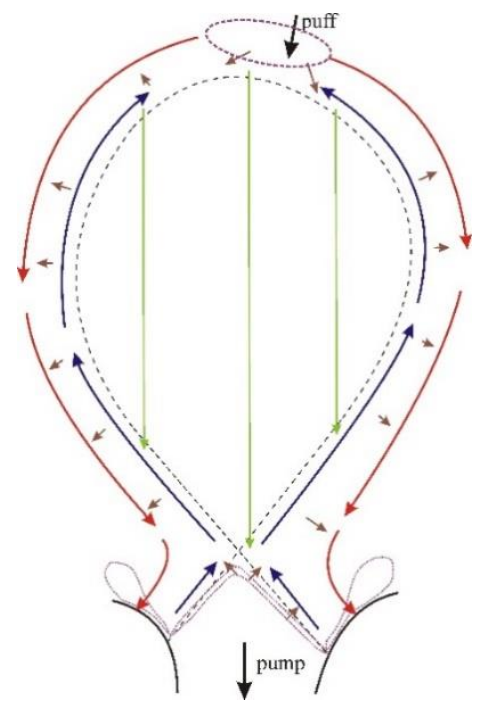

Fig.9. Schematic illustration of the ITER SOL main ion poloidal flow pattern. Violet dashed lines mark zones of ionization. Red/blue arrows represent poloidal flows towards and away from the divertors respectively. Brown arrows represent the direction of diffusive radial fluxes and green arrows that of the grad-B drift. 


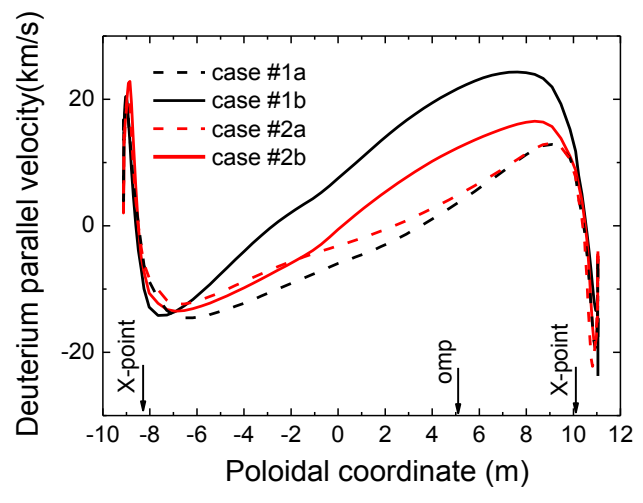

(a)

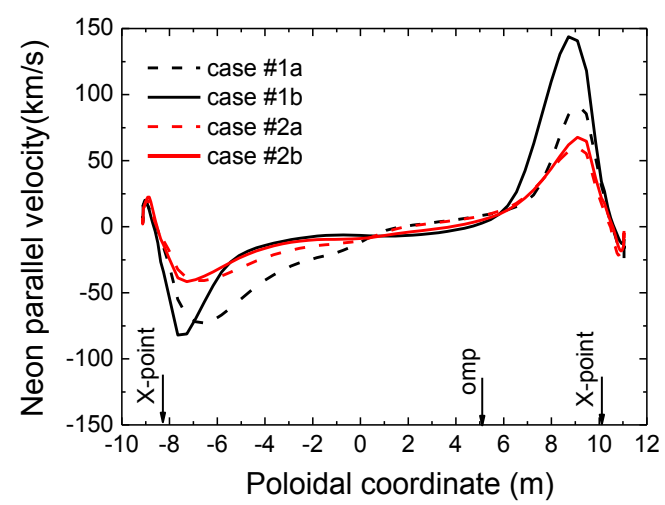

(c)

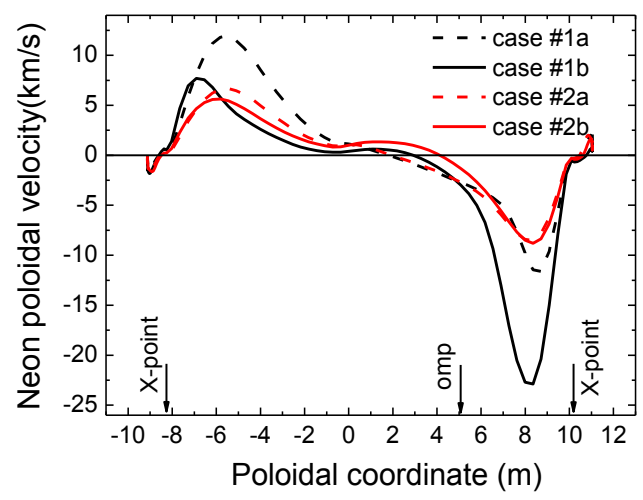

(e)

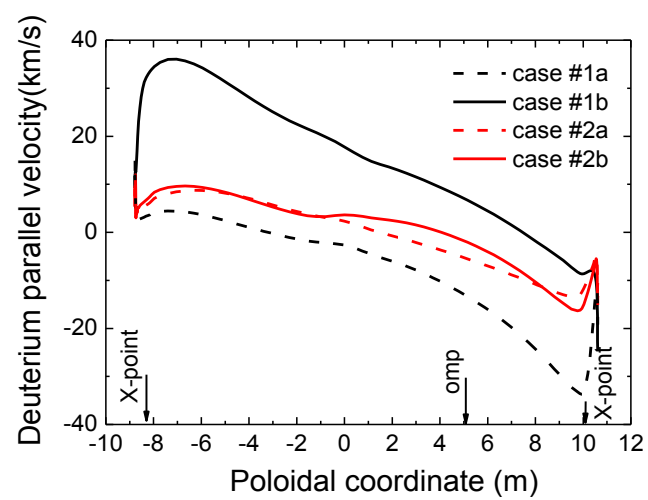

(b)

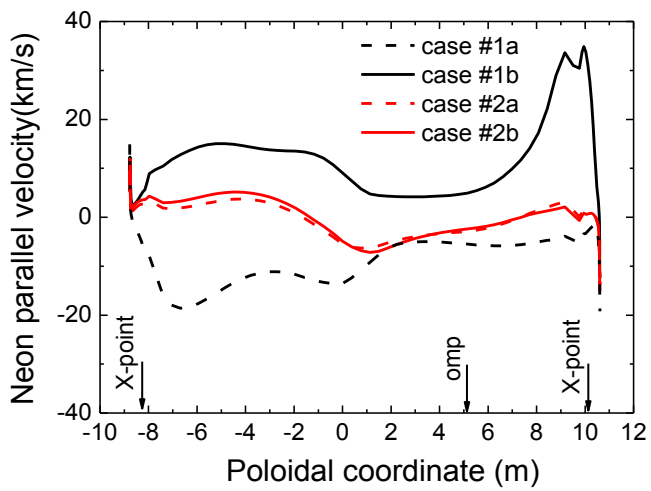

(d)

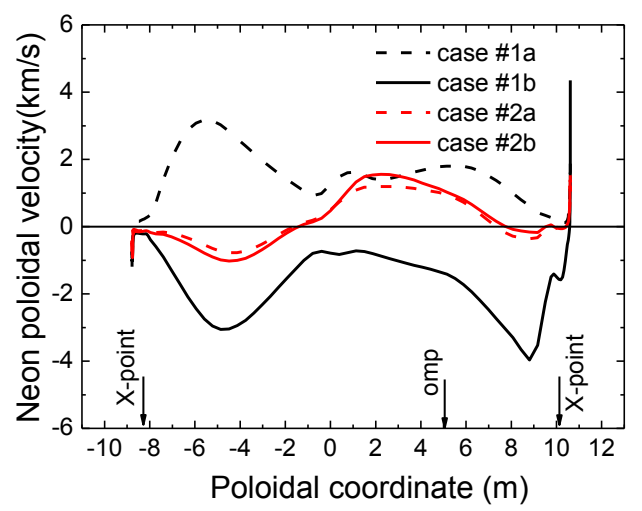

(f)

Fig.10. Poloidal distribution of the parallel velocity (positive velocity directed towards inner target) of (a) main ions in the near SOL, $\left(r-r_{\text {sep }}\right)_{o m p}=2 \mathrm{~mm}$; (b) main ions in the far SOL $\left(r-r_{\text {sep }}\right)_{o m p}=22 \mathrm{~mm}$; (c) $\mathrm{Ne}$ in the near SOL, $\left(\mathrm{r}-\mathrm{r}_{\mathrm{sep}}\right)_{\mathrm{omp}}=2 \mathrm{~mm}$; and (d) Ne in the far SOL, $\left(\mathrm{r}-\mathrm{r}_{\mathrm{sep}}\right)_{\mathrm{omp}}=22 \mathrm{~mm}$. (e) poloidal velocity (positive velocity directed towards outer target) of $\mathrm{Ne}$ in the near SOL, $\left(\mathrm{r}-\mathrm{r}_{\mathrm{sep}}\right)_{\mathrm{omp}}=2 \mathrm{~mm}$ and (f) in the far SOL, $\left(r-r_{\text {sep }}\right)_{o m p}=22 \mathrm{~mm}$ 


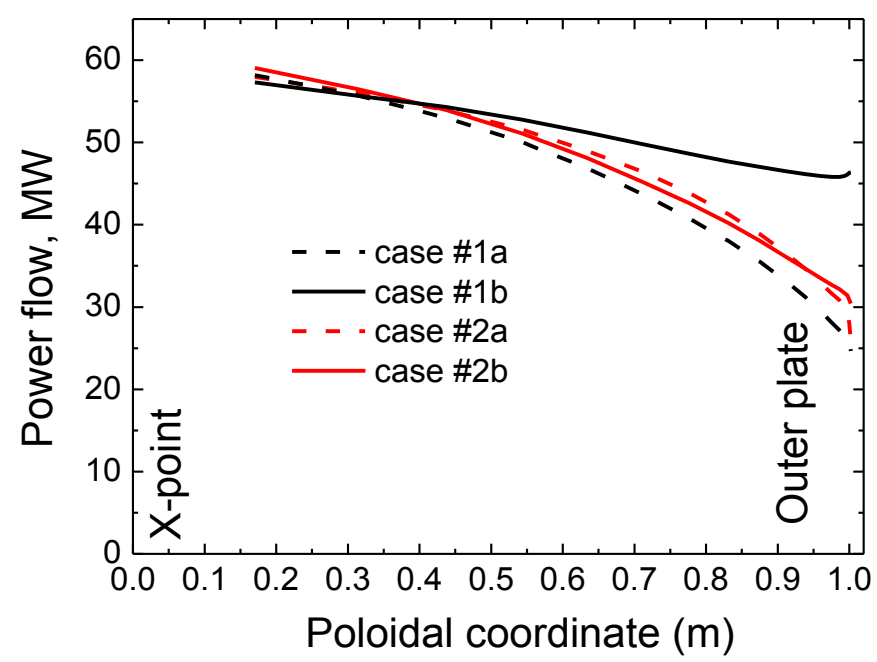

Fig.11. Poloidal flow of the plasma power (including the ionization energy carried by ions) towards the outer target, integrated over the radial coordinate for Cases \#1 and 2.

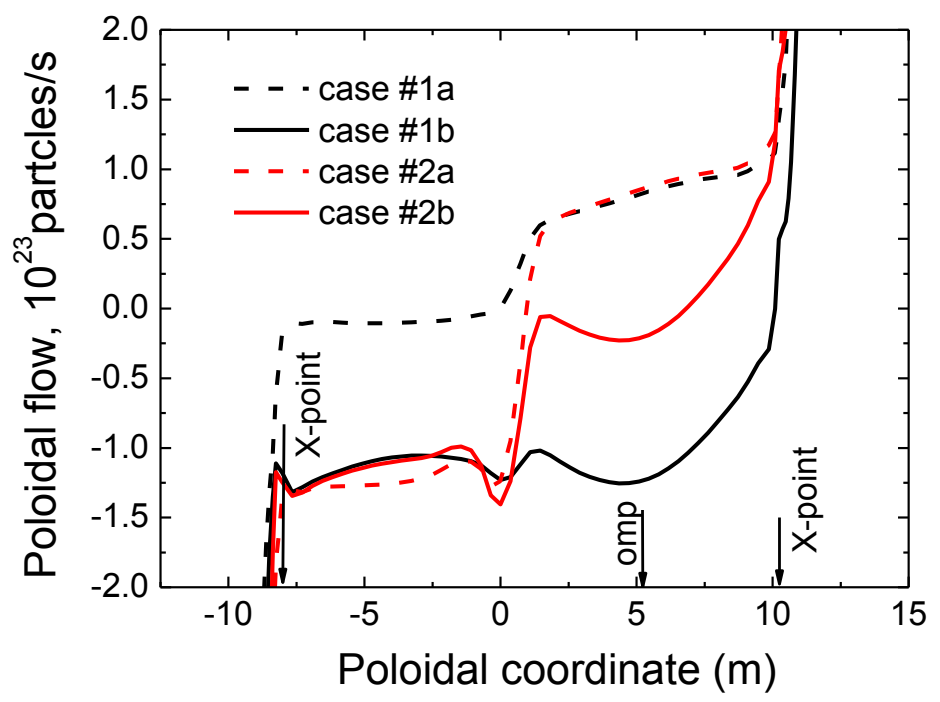

Fig.12. Poloidal flow of $\mathrm{D}$ ions (sum of the poloidal projections of the parallel flow and poloidal $\mathrm{E} \times \mathrm{B}$ flow) through the SOL integrated over the radial coordinate for Cases \#1 and 2. 


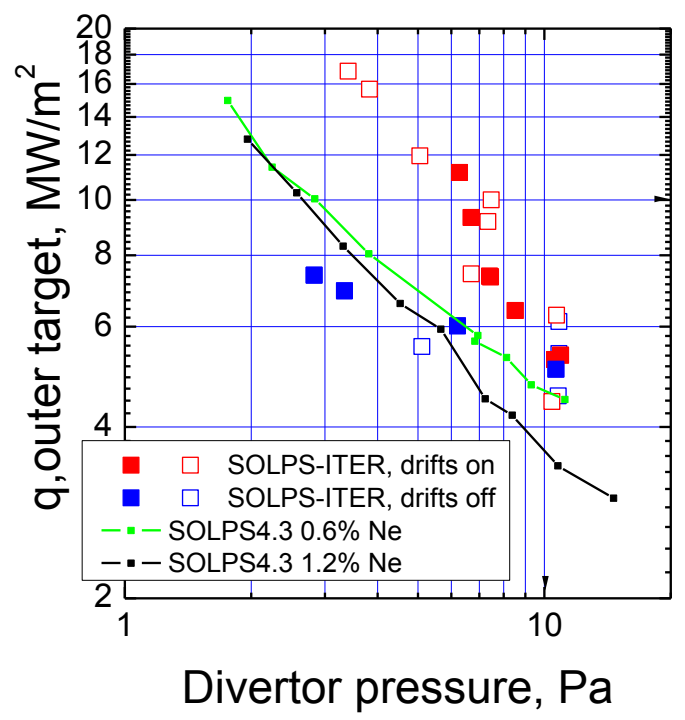

Fig. 13. Operating range for the peak outer target power load as a function of the neutral divertor pressure from SOLPS-ITER with and without drifts (filled points at $\mathrm{c}_{\mathrm{Ne}}=0.8-1.1 \%$, all points in the range $\left.\mathrm{c}_{\mathrm{Ne}}=0.35-2 \%\right)$ and from the SOLPS-4.3 database at two different values of $\mathrm{c}_{\mathrm{Ne}}$.
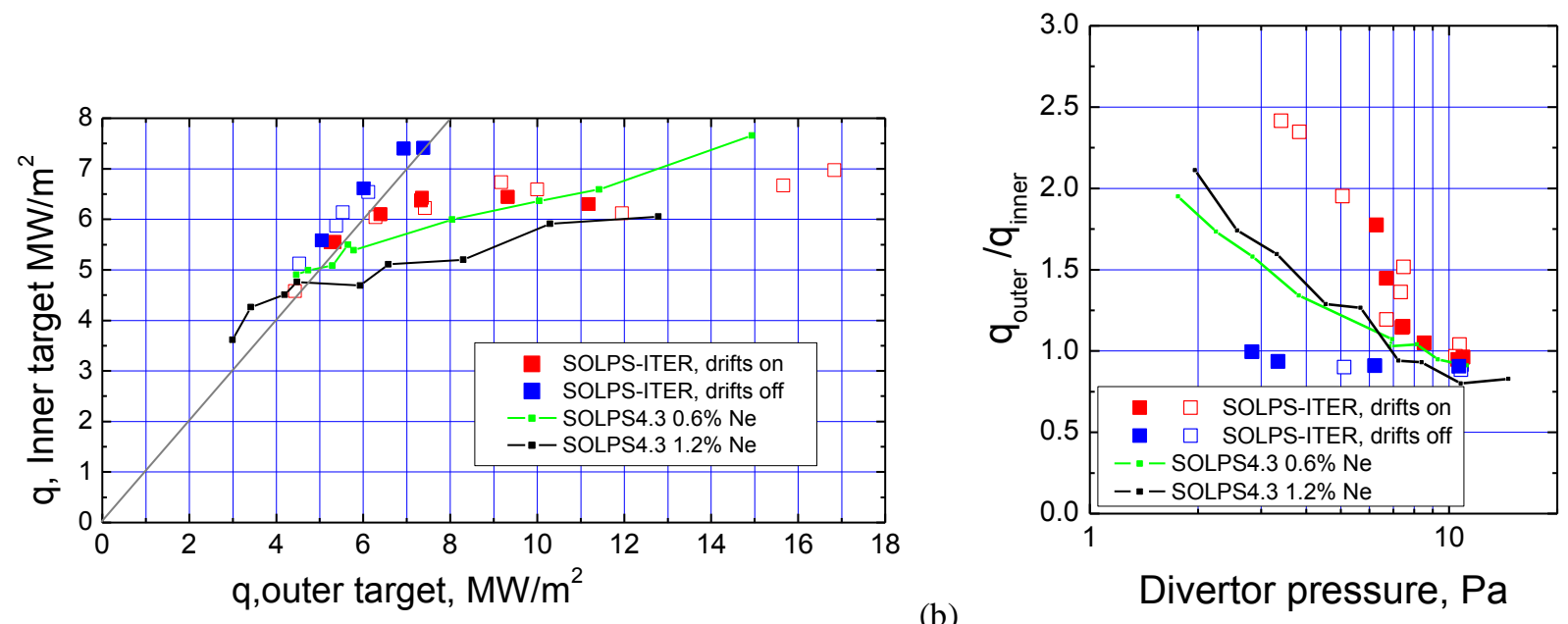

(a)

(b)

Fig.14. (a) Inner versus outer target peak power loads for the same datasets as in Fig. 13; grey line corresponding to 1:1 asymmetry; (b) rate of outer to inner target peak power load versus neutral divertor pressure 